\title{
Development of a reliable and simple pressure wave generator for pulse tube refrigerators
}

\author{
R. Karunanithi, ${ }^{\text {a) }}$ S. Jacob, S. Kasthurirengan, Upendra Behera, and D. S. Nadig \\ Centre for Cryogenic Technology, Indian Institute of Science, Bangalore 560012, India
}

\begin{abstract}
The pressure wave generator is a critical component of a pulse tube refrigerator. Conventionally either a solenoid operated electromagnetic valve or a rotary valve driven by a hermetically sealed motor is used for this. In this article, we present a novel pressure wave generator design based on cam operated valves for use with pulse tube refrigerators. This, when coupled with an inverter drive (variable frequency drive), forms a versatile pressure wave generator in the range between 1 and 10 Hz. In a single stage double inlet pulse tube using this type of pressure wave generator, refrigeration power of about $6 \mathrm{~W}$ at $77 \mathrm{~K}$ and a no load temperature of about $42 \mathrm{~K}$ were obtained. The above results are comparable to those obtained with pulse tube systems based on other two types of pressure wave generators. While the rotary valve based pressure wave generator is prone to developing leaks at the rotating seal, the solenoid valve based system cannot be operated continuously due to coil failure. The cam operated valve based pressure wave generator overcomes the above shortfalls of rotary valve and solenoid valve type pressure generators and is well suited for continuous operation of the refrigerator.
\end{abstract}

\section{INTRODUCTION}

For over two decades in the area of pulse tube refrigerator (the basic pulse tube discovered by Gifford and Longsworth $^{1}$ ) there have been significant advancements. Due to the absence of a mechanical moving component at the cold side of the system, pulse tube refrigerators find applications in semiconductor fabrication, charge coupled devices for astronomical telescopes, super conducting quantum interference device (SQUID) detection for nondestructive tests, and medical applications where vibration and noise should be very low and the system should have high reliability. A schematic of a double inlet pulse tube refrigerator (DIPTR) is shown in Fig. 1. The system without a bypass valve, orifice valve, and reservoir forms a basic pulse tube in which the hot end of the pulse tube is closed. The addition of a reservoir and orifice valve converts it into an orifice pulse tube. ${ }^{2}$ In the double inlet pulse tube, ${ }^{3}$ there is a bypass valve between the regenerator and the pulse tube at the room temperature end. A medium pressure (17-20 bar) helium compressor and the pressure wave generator produce the pressure wave required. The regenerator contains a porous matrix which absorbs heat from the gas when it goes towards the pulse tube and gives it off when the gas flows in the opposite direction. The pulse tube is an empty tube with heat exchangers (HEs) (hot end HE and cold end HE) at both the ends. In basic pulse tube mode, when the system is subjected to oscillating gas flow, due to a thermoacoustic effect (an acoustically driven temperature effect), the open end of the pulse tube is cooled and heat is transported to the hot end where it is rejected in the HE. In the orifice pulse tube intro- duction of a needle valve (orifice) and a buffer volume (reservoir) enhance performance. This arrangement creates the required phase shift between the pressure wave and the mass flow at the warm end, so that they are in phase at the cold end. Introduction of the bypass valve (double inlet pulse tube) improves the performance further by sending part of the gas directly to the warm end of the pulse tube, thus reducing mass flow of the gas through the regenerator. One of the critical components of the pulse tube is the pressure wave generator. Usually a solenoid operated or a rotary valve operated pressure wave generator is used in pulse tube

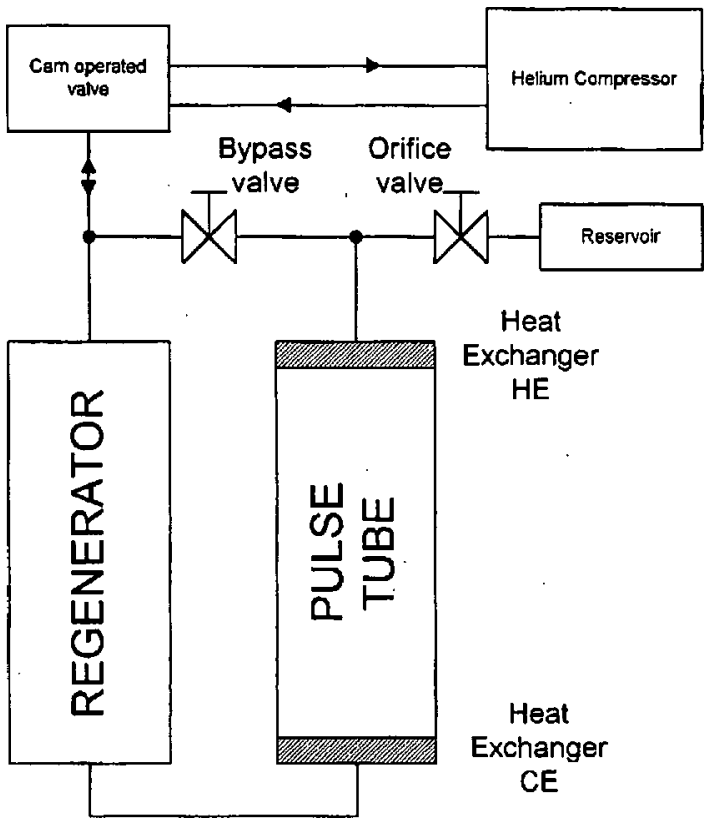

FIG. 1. Schematic of the double inlet pulse tube refrigerator. 


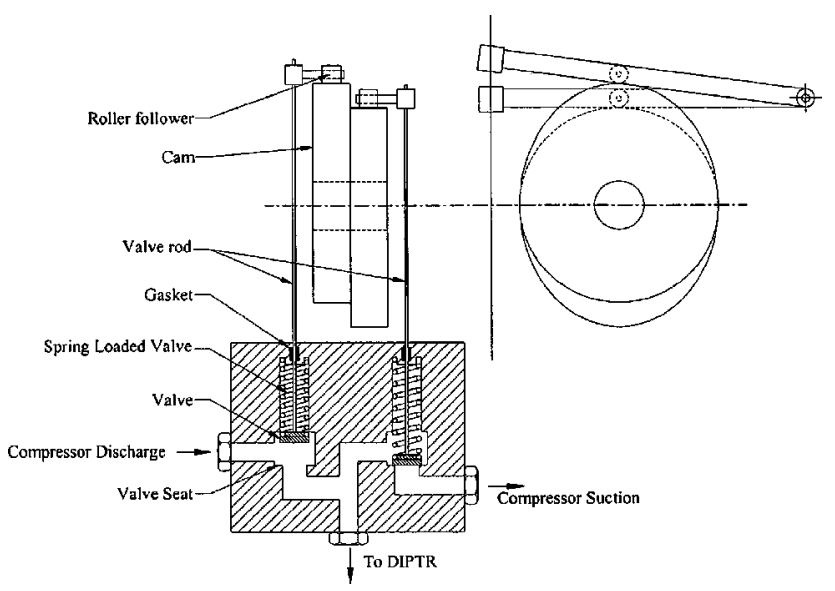

FIG. 2. Schematic of the cam operated pressure wave generator.

systems. ${ }^{4}$ While the solenoid operated pressure wave generator is prone to failure beyond a limited number of operations, the rotary valve system is likely to develop leaks in the seal between the high/low pressure inlets and the outlet. For commercialization of the pulse tube system and for reliable continuous operation for a very long period without failure, the above problems have to be solved. In this article, we describe a novel pressure wave generator based on cam operated valves for use with pulse tube refrigerators that does not have the above problems and experimental results of the DIPTR thus developed.

\section{DESCRIPTION}

The pulse tube refrigerator is similar in operation to the Gifford-McMahan cryocooler (GM cooler); except that the oscillating gas piston replaces the mechanical displacer of GM cooler. Figure 1 shows a schematic of the pulse tube refrigerator that consists of a regenerator $[19 \mathrm{~mm}$ outer diameter (o.d.), $1 \mathrm{~mm}$ wall thickness, and $210 \mathrm{~mm}$ length stainless steel (SS) tube] filled with $\sim 1800$ circular disks of 200 mesh stainless steel wire screens and a stainless steel pulse tube (19 mm o.d., $1 \mathrm{~mm}$ wall thickness, and $250 \mathrm{~mm}$ length). Both of them are kept inside a vacuum vessel. The other components of the pulse tube refrigerator are a helium compressor, double inlet and orifice needle valves (a Hoke metering valve), a pressure wave generator, and a reservoir ( $\sim 500 \mathrm{ml}$ capacity). It is a device for producing cryogenic temperatures. It is a closed system that makes use of pressure pulses generated by the compressor and the pressure wave

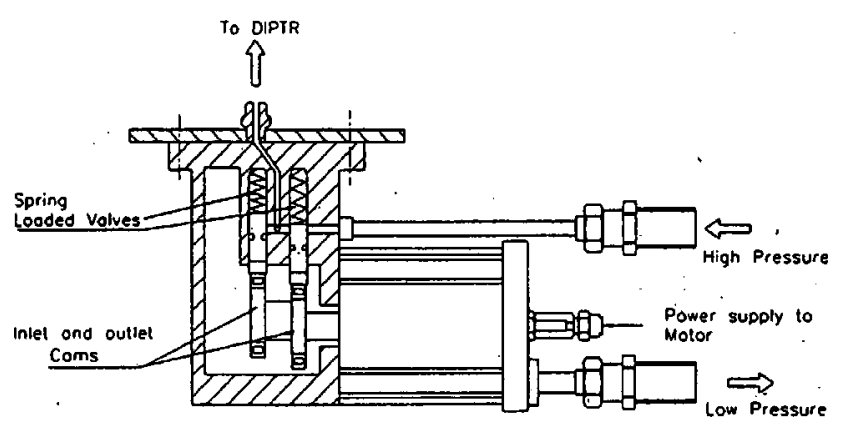

FIG. 3. Schematic diagram of the cross section of the cam operated pressure wave generator.

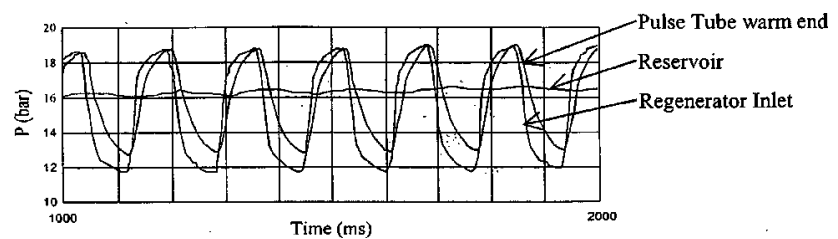

FIG. 4. Pressure wave patterns at the regenerator inlet, warm end of pulse tube, and at the orifice of the double inlet pulse tube.

generator to send oscillating gas flow into the rest of the system. This helium gas flow can carry the heat away from a low temperature point (cold end $\mathrm{HE}$ ) if the conditions are right.

In this article, a novel concept of pressure wave generation for a pulse tube refrigerator is reported. A cam operated spring-loaded valve based pressure wave generator is presented in the work. A schematic of the design is shown in Fig. 2. It consists of a set of spring-loaded valves coupled to the suction and discharge sides of compressor. The outlets of both valves are coupled together to form a common inlet to the DIPTR. A set of valve rods couples the valves to the cam mechanism. The rods are always kept under tension to avoid leaks at the valve seat. The cams can be rotated at varying speeds using a motor coupled to a variable frequency drive. Similar cam operated systems are employed in the expansion engine of a Collins helium liquefier to connect high and low pressure gas streams to the expansion engine. But to our knowledge, such a system has not been employed as a pressure wave generator for pulse tube refrigerators elsewhere. Instead of developing such a system, if one has access to the cold head drive of a commercial GM cryocooler, it can be suitably modified and used as a pressure wave generator for a pulse tube refrigerator. The cold head drive performs two functions in the GM cryocooler. It converts circular motion of the motor to reciprocal motion to move the stem up and down to connect the integral regenerator displacers of the two stages. This is done in synchronization with opening and closing of the high- and low-pressure sides of the compressor by operating the corresponding cam operated spring-loaded valves. For pulse tube application, since there is no mechanical displacer in the system, it is sufficient if only the pressure wave is generated by using the cam operated spring-loaded valves. Hence, the components needed to move the integral regenerator displacer up and down are discarded from the cold head drive in the present system and only the parts needed to generate the pressure wave are retained. A schematic of the modified pressure wave generator is shown in

TABLE I. Comparison of pressure data of the cam operated system and the rotary valve system.

\begin{tabular}{lcc}
\hline \hline Parameters & $\begin{array}{c}\text { Cam operated pressure } \\
\text { wave generator }\end{array}$ & $\begin{array}{c}\text { Rotary valve based pressure } \\
\text { wave generator }\end{array}$ \\
\hline$P_{\max }$ & $17 \mathrm{bar}$ & $14.5 \mathrm{bar}$ \\
$P_{\min }$ & $9.75 \mathrm{bar}$ & $10 \mathrm{bar}$ \\
$\Delta P$ & $7.25 \mathrm{bar}$ & $4.5 \mathrm{bar}$ \\
$P_{\max } / P_{\min }$ & 1.75 & 1.45 \\
$P_{\text {average }}$ & $13.5 \mathrm{bar}$ & $12 \mathrm{bar}$ \\
\hline \hline
\end{tabular}

${ }^{\text {aAfter Ref. } 4 .}$ 


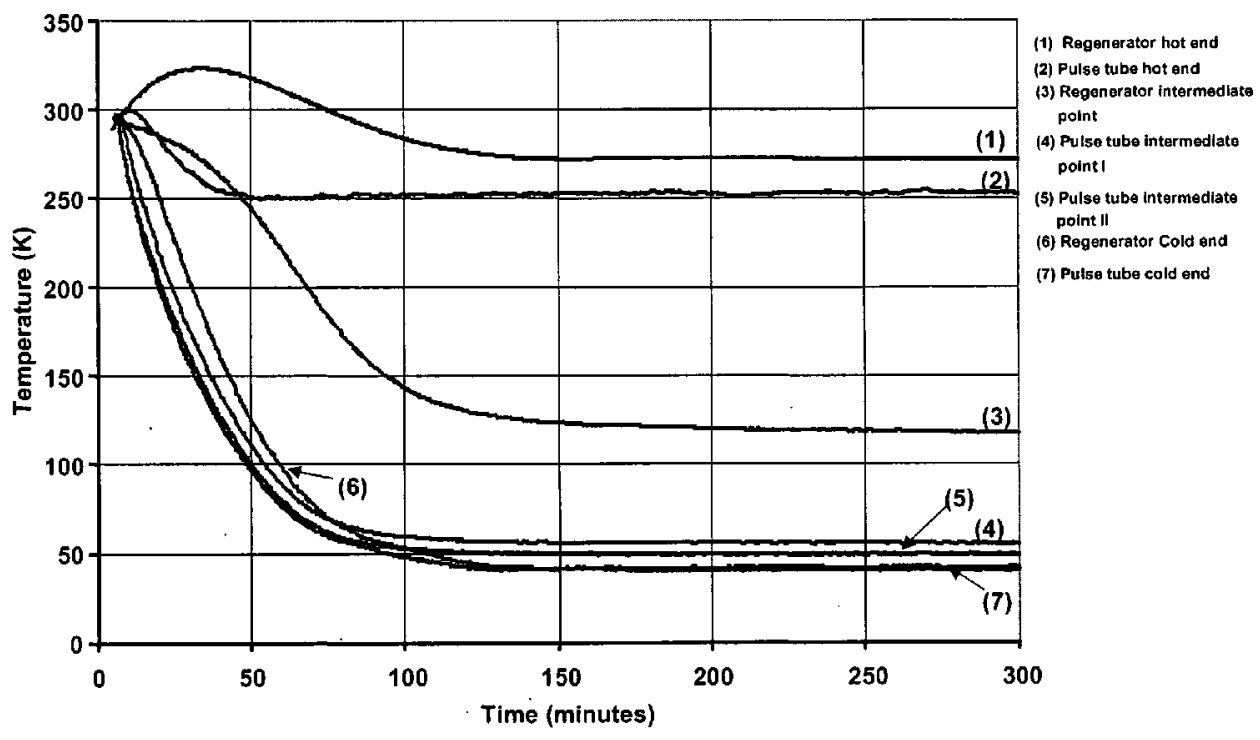

FIG. 5. Cool down profile of the double inlet pulse tube refrigerator.

Fig. 3. The compressor discharge and suction sides are coupled to the device with the help of quick disconnect coupling (Aeroquip coupling). Common output from the device is connected to the warm end of the regenerator. Since it is necessary to change the frequency of operation to maintain the pressure wave in phase with the mass flow at the cold end of the pulse tube to get maximum refrigeration power a variable frequency drive of approximately $750 \mathrm{~W}$ is used to operate the cold head drive motor. By varying the frequency of the drive, it is possible to change the operating range of pressure wave generated from 1 to $10 \mathrm{~Hz}$. It is also possible to vary the duty cycle of high- and low-pressure profiles by adjusting the cam setting. For a set of parameters such as openings of the orifice and double inlet valves, dimensions of the components, etc., it is necessary to find the optimum frequency of operation to get the maximum refrigeration capacity. Similarly, by keeping the duty cycle at different values, the system can be fine-tuned. Figure 4 shows the pressure wave patterns at the inlet to the regenerator, pulse tube warm end, and at the buffer volume of the pulse tube. Due to the cam operated valves, the suction and discharge sides are alternatively connected to the common outlet instantaneously. Hence, the wave form is close to that of a square wave at the regenerator inlet. The pressure amplitude is maximum at this point since it represents the total mass flow into the system. At pulse tube hot end, it is slightly less than that at the regenerator due to the drop in pressure in the regenerator. In the buffer, it is very close to the mean value as the gas is allowed into the buffer through a high impedance orifice valve. The phase of the oscillating pressure wave is adjusted with the help of the orifice and double inlet valves such that it is in phase with the mass flow at the cold end.
The results of the cam operated system developed by us are compared to those of a rotary valve based system ${ }^{4}$ in Table I. From the data, it can be concluded that the performance of this system is comparable to that of a rotary valve based system.

Typical cool down characteristics of the pulse tube system using the above pressure wave generator are shown in Fig. 5. The temperature data from seven locations in the pulse tube system are monitored. It can be observed from Fig. 5 that the cold end of the pulse tube and regenerator reaches a steady state value of about $42 \mathrm{~K}$ in approximately about $1 \mathrm{~h}$. The temperature increase per watt of heat load is about $8 \mathrm{~K}$ and the refrigeration power at $77 \mathrm{~K}$ is about $6 \mathrm{~W}$. The system has been in operation for the past three years, with each test run exceeding continuous operation for five days. There has been no failure of the pressure wave generator during the tests up to now. From the results, it can be concluded that the cam operated pressure wave generator performs very well and has the added advantages of better durability and reliability.

\section{ACKNOWLEDGMENT}

The authors acknowledge financial support by the Indian Institute of Science-ISRO Space Technology Cell for this work as a project under STC.

${ }^{1}$ W. E. Gifford and R. C. Longsworth, Trans. ASME, J. Eng. Ind. 86, 264 (1964).

${ }^{2}$ E. I. Mikulin, A. A. Tarasov, and M. P. Shkrebyonock, Advances in Cryogenic Eng. (Plenum, New York, 1984), Vol. 29, p. 629.

${ }^{3}$ S. Zhu, P. Wu, and Z. Chen, Cryogenics 30, 514 (1990).

${ }^{4}$ J. Liang, Ph.D. thesis, Institute of Engineering Thermophysics, Chinese Academy of Sciences, 1993, p. 145. 